\title{
Towards Automated Surgical Documentation using automatically generated checklists from BPMN models
}

\begin{abstract}
The documentation of surgeries is usually created from memory only after the operation, which is an additional effort for the surgeon and afflicted with the possibility of imprecisely, shortend reports. The display of process steps in the form of checklists and the automatic creation of surgical documentation from the completed process steps could serve as a reminder, standardize the surgical procedure and save time for the surgeon. Based on two works from Reutlingen University, which implemented the creation of dynamic checklists from Business Process Modelling Notation (BPMN) models and the storage of times at which a process step was completed, a prototype was developed for an android tablet, to expand the dynamic checklists by functions such as uploading photos and files, manual user entries, the interception of foreseeable deviations from the normal course of operations and the automatic creation of OR documentation.
\end{abstract}

Keywords: checklists, BPMN, OR documentation, cochlear surgery

\section{https://doi.org/10.1515/cdbme-2021-1029}

\footnotetext{
*Corresponding author: Elisaveta Just: Reutlingen University, School of Informatics, Research Group Computer Assisted Medicine (CaMed), Alteburgstraße 150, 72762 Reutlingen, Germany,

E-mail: Elisaveta.Just@Student.Reutlingen-University.DE Katharina Schaumann: Dept. of Otorhinolaryngology, University Hospital Düsseldorf, Moorenstr. 5, 40225 Düsseldorf, Germany

Denise Junger: Reutlingen University, School of Informatics, Research Group Computer Assisted Medicine (CaMed), Alteburgstraße 150, 72762 Reutlingen,Germany Claudia Ryniak: Reutlingen University, School of Informatics, Research Group Computer Assisted Medicine (CaMed), Alteburgstraße 150, 72762 Reutlingen, Germany

Thomas Klenzner: Dept. of Otorhinolaryngology, University Hospital Düsseldorf, Moorenstr. 5, 40225 Düsseldorf, Germany

Oliver Burgert: Reutlingen University, School of Informatics,

Research Group Computer Assisted Medicine (CaMed),

Alteburgstraße 150, 72762 Reutlingen, Germany
}

\section{Introduction}

This paper describes the development of a user interface that allows the representation of dynamic checklists for surgical procedure steps and the automatic creation of surgical documentation from them. This can provide a memory aid for the surgeon, prevent errors in the OR, and simplify the documentation of surgeries.

Most checklists available today contain only a few superficial process steps [1-2] or are limited to mostly linear intervention courses [3]. However, surgery is a complex process and does not always proceed in the same way. A dynamic checklist, which contains different selection options, the ticking of which leads to further matching checklist elements [4], could represent such a complex procedure. Timestamps, at which the process steps were marked as completed, could be stored, which would simplify the documentation of an operation [5]. For this purpose, we developed a dynamic checklist tool that converts BPMN process models for specific surgery into a checklist $[4,6]$. In this system, decision nodes, which define the following intervention course, are integrated. After an answer at a decision node is selected, associated checklist items are loaded.

The documentation of an operation is usually not technically supported and is created by surgeons from memory after the operation. However, since important steps are sometimes omitted in the process [7], some projects support the documentation of surgeries by video recordings [8-9], which can supplement the documentation after surgery and thus lead to more accurate documentation [9]. Despite video recording, documentation must be manually written by the surgeon after surgery, which requires additional time. To facilitate documentation, Killer developed a system that allows process steps and the times at which they were completed to be stored intraoperatively [5]. Since the system is only adapted to linear procedures, it would be useful to combine it with the dynamic checklists of Ryniak [4] described earlier. The goal of this work is to create such a combination. A prototype, which generates checklists from cochlear implantation BPMN 
models was developed. The prototype should offer the possibility to upload and display files from the file system, take photos, and make manual entries. During surgery, while performing certain steps, predictable events could occur. Such events must also be documented and may entail further steps. All checked elements, which represent performed surgical procedure steps, should automatically be included, into the surgical documentation as full sentences. Additionally the documentation should contain the time stamps, at which the corresponding steps were checked.

\section{Methodology}

The cochlear implantation was modeled as a BPMN process model. The basis for the process model was a document that contained the cochlear surgery process in bullet points, which was used internally at the university hospital of the Heinrich Heine University of Düsseldorf. The document verbally described the surgical steps to be taken, and possible changes in the intervention course, as well as foreseeable events. The resulting BPMN model was created in an iterative modeling process between the clinical and the technical project partners. To upload files, take photos, or for manual input, additional BPMN activities were added, namely"upload file", "take photo" and "enter $[\ldots]$ ".

Predictable events were modeled as events in the BPMN model. As shown in Figure 1, a signal-receiver event is attached to a process step. After that, further process steps can follow.

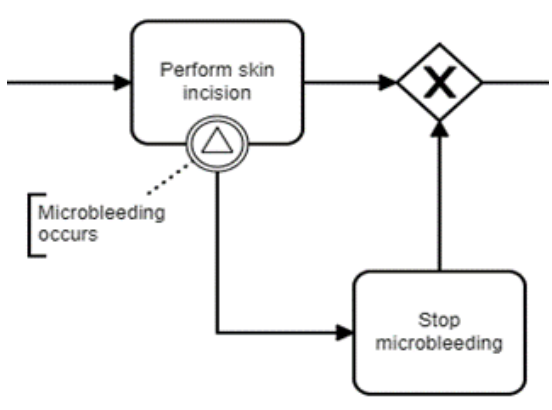

Figure 1: Predictable Event during cochlear surgery in BPMN

At some points, XOR-gateways are placed, whose outgoing branches lead to several different progressions.

The BPMN elements are to be displayed on the user interface of the prototype in a way that is comprehensible to the user. For this purpose, wireframes were used to plan how the BPMN elements would later be displayed on the user interface. The transformation of the BPMN elements into wireframe elements is shown in Table 1. In [6], we introduced the transition from the start and end events, all activities except "upload file", "take photo" and "enter [...]", parallel branches, XOR branches, text annotations, and ordinary sequence flows. This transition concept was extended in this work to include intermediate events and activities to upload files, take photos, and manual inputs. XOR-gateways now no longer represent yes/no questions, as was the case in [6], but multiple-choice fields.

Table 1: Design Concept Checklist Tool

\begin{tabular}{|c|c|}
\hline $\begin{array}{l}\text { BPMN } \\
\text { element }\end{array}$ & Wireframe Element \\
\hline Subprocess & Checklist \\
\hline Activity & Checklist task \\
\hline $\begin{array}{l}\text { Start- and } \\
\text { End-event }\end{array}$ & Heading \\
\hline $\begin{array}{l}\text { AND- } \\
\text { gateway }\end{array}$ & $\begin{array}{l}\text { Like Sequenceflow, no influence on the } \\
\text { order }\end{array}$ \\
\hline $\begin{array}{l}\text { XOR- } \\
\text { gateway }\end{array}$ & $\begin{array}{l}\text { Indentation, dynamic loading depending } \\
\text { on the selection }\end{array}$ \\
\hline Sequence flow & Order, priority \\
\hline $\begin{array}{l}\text { Text } \\
\text { annotation }\end{array}$ & $\begin{array}{l}\text { Further information for a specific } \\
\text { element }\end{array}$ \\
\hline $\begin{array}{l}\text { Activity } \\
\text { "upload data" }\end{array}$ & $\begin{array}{l}\text { Symbol to select and upload data (only } \\
\text { txt-files supported) }\end{array}$ \\
\hline $\begin{array}{l}\text { Activity "take } \\
\text { photo" }\end{array}$ & Symbol to take a picture \\
\hline $\begin{array}{l}\text { Activity } \\
\text { "[enter] }]\end{array}$ & $\begin{array}{l}\text { Input fields to enter data, the label is } \\
\text { determined by the name of the activity }\end{array}$ \\
\hline Signal event & $\begin{array}{l}\text { Predictable events are shown as text of } \\
\text { radio buttons in a box, if selected the } \\
\text { respective following tasks are shown }\end{array}$ \\
\hline
\end{tabular}

\section{Results}

\subsection{Data and experimental Setup}

The developed prototype is a web application based on the client-server model. The application was primarily developed for use on an Android tablet but can also be displayed on other screen sizes and devices. 


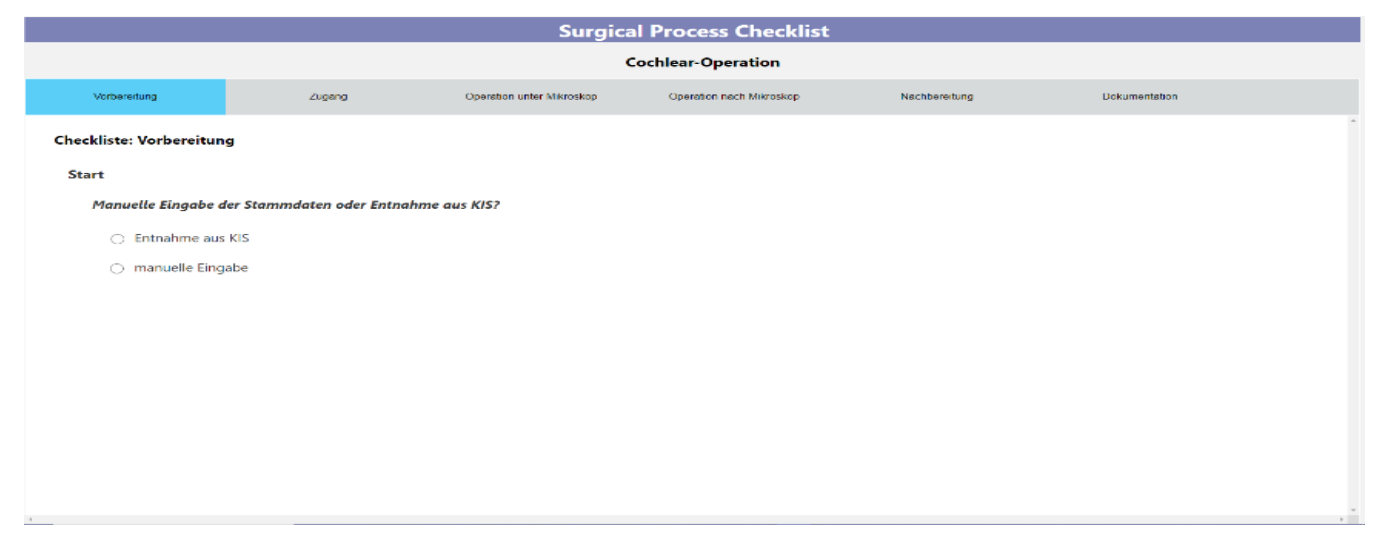

Figure 1: GUI of the Checklist Tool

The system algorithm parses xml-files and converts BPMN elements into corresponding Graphical User Interface (GUI) symbols. First, the "startEvent" tag is picked out and added accordingly on the GUI. Then, the tags "outgoing" and "incoming" are used to search for the subsequent elements. A distinction was made between activities, XOR-gateways, AND-gateways, text annotations and start- and endevents. [4] These elements were converted into elements of the GUI according to Table 1. The GUI design can be seen in Figure 2. Case distinctions have been added to the activities. If the activity has the name "Upload File", a txt-file from the filesystem can be selected for upload (see Figure 3).

Manuelle Eingabe der Stammdaten oder Entnahme aus KIS?

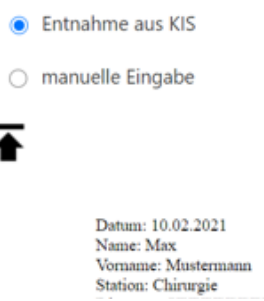

Figure 2: Data Upload Checklist Tool

If the following activity contains the word "enter", input fields appear which the user can fill in.

If the following activity is called "take photo", the camera view will appear on the GUI. If a photo is taken, it will be displayed below the camera view on the GUI. The photo will then be downloaded automatically.

After an activity has been checked, a function checks whether an event is attached. If this is the case, a box appears for each event (see Figure 4), in which the text annotation of the event is displayed as a radio button. If this is selected, all subsequent steps of the path are displayed until they join the main path. Several activities can be contained in the additional path.

In the "Documentation" tab, the documentation created automatically up to that point is displayed in a text field (see Figure 6). Here, uploaded files, manual entries, additional activities that were caused by events and all other checklist elements that have already been checked are displayed as full sentences with corresponding timestamps.

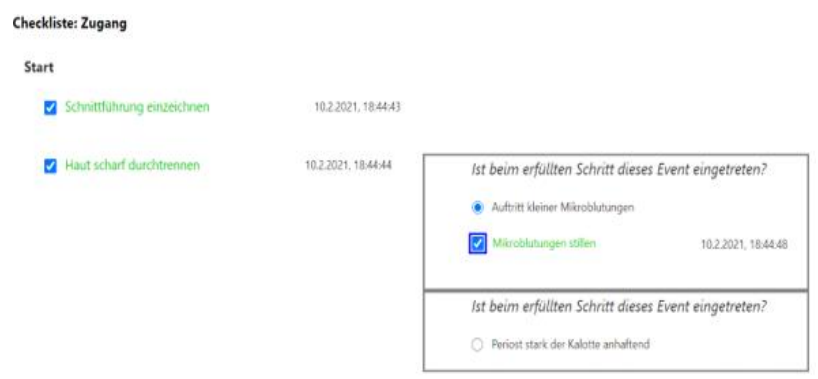

Figure 4: Predictable event shown within the Checklist Tool

It is also possible to define several full sentences per activity. In this case, the first of the possible sentences is displayed in the "Documentation" tab in the text field. By clicking on a wrench symbol a sentence selection window is displayed (see Figure 5). The selected sentence is then displayed in the text field at the appropriate position. In the "Documentation" tab, the operator can also edit the documentation and save it as a txt-file. All images taken during documentation are displayed in the "Documentation" tab below the text field. However, these must be inserted manually into the documentation.

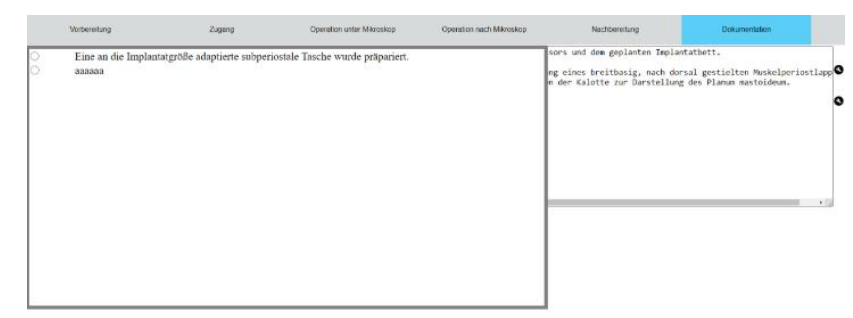

Figure 5: Sentence selection Checklist Tool 


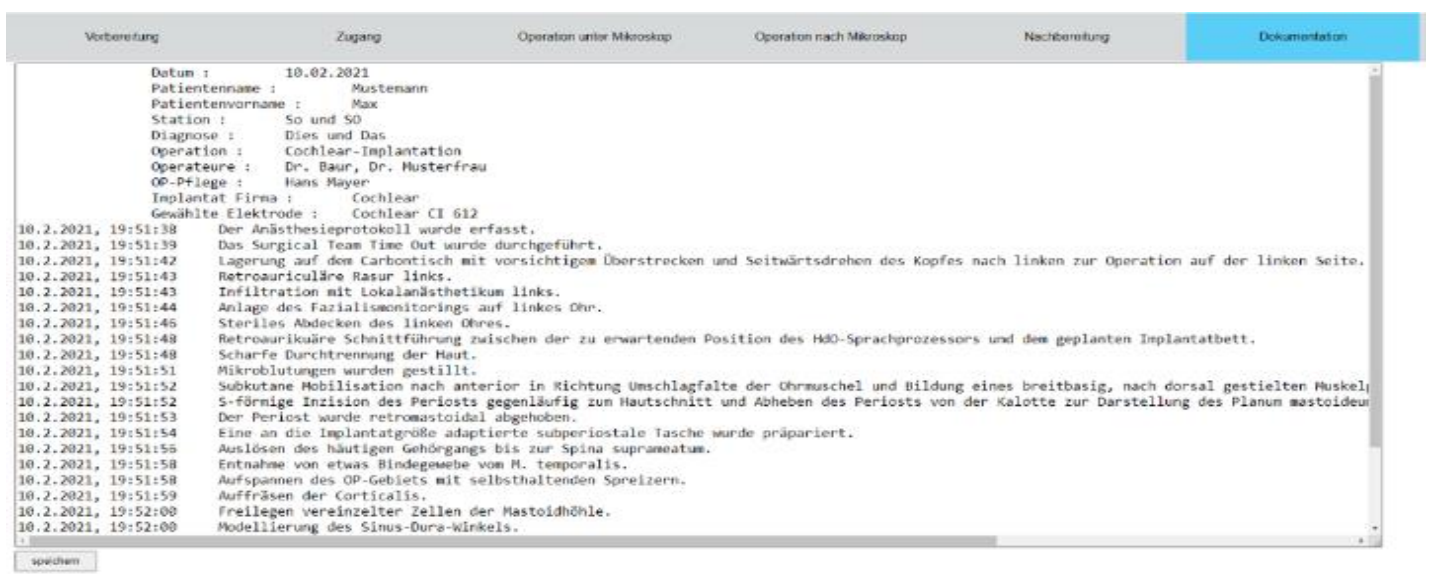

Figure 6: Documentation tab oft he Checklist Tool

\subsection{Usability test}

The system was tested by one surgeon at the clinic of the Heinrich Heine University Düsseldorf. The remote usability test was performed on a laptop, in a non-sterile environment, on a petrous bone model. During the test, some possible extensions to the system were expressed. For example, the view and control of the checklist could be possible on the microscope or through a head-up display, so that the surgeon would not have to constantly switch between microscope and checklist. The division into user groups would also make sense. In this way, more small steps could be displayed for trainees, while the procedure for experienced surgeons would only be divided into rough steps. In addition, a way to display XOR-gateways in any order would also be desirable. This is currently not possible, because the subsequent steps of a branch are only displayed after the selection of an answer to the XOR-gateway question. A further topic was the handling of unforeseen events. Here, the input of additional steps or texts or voice recordings would be useful for a sub-process. Linking the system to MEDICO and DICOM, as well as to the technician's computer would also be useful.

Also, some steps were not intuitive. For example, it was not always clear that the sub-process was not yet finished when unselected XOR-gateways were displayed, which is why no subsequent steps were displayed. In the course of the test, however, the test person also noticed that all sub-processes were ended with the heading "End", which was then considered helpful. There were also a few errors in the modeling, as some steps were missing. Besides, it was not clear that manual entries must be confirmed by clicking the Enter key.

The fact that the process can be run in any order was noticed as positive. The ability to make manual user entries and take photos was also found to be useful. The automated creation of documentation was considered useful and an easement in the daily clinical routine. Overall, the tester found the system to be non-distracting and quick to use. She also said that she would like to use it, but that there was the hurdle of the timeconsuming preparation of the input device.

In further versions of the tool the discovered errors should be fixed, the not intuitive elements should be improved and the suggested additional functionalities should be added.

\section{Discussion}

The prototype created in this work represents a memory aid for the surgeon through the display of surgical steps and facilitates his daily routine through the automated creation of documentation. With the help of XOR-gateways, events, and manual user input, a complex surgical procedure can be displayed and supported. The surgeon also has the option of taking photos during certain steps and loading txt-files into the system. These can supplement the automatically generated documentation. The checked steps are displayed as full sentences as part of the automatically generated documentation, in the order in which they were performed and with the time at which they were checked. We have to emphasize that in clinical routine the timestamps migh not represent the real time spent on a process step. Especially in emergency situations, it might be possible that several steps are performed and afterwards all steps are checked. Therefore the timestamps should just be interpreted as odering of surgical procedure steps. This documentation can be edited within the system by the operator and saved as a txt-file. A tablet was chosen as the input device, which allows display in the sterile field close to the surgical site. 
This work offers some further development possibilities that could be implemented in the future. No usability tests with several users or clinical tests have been performed so far. These should be done in the future. The documentation is saved unformatted as a txt-file. Saving formatted documentation in Word and a connection to MEDICO could be implemented in further projects. A connection to the HIS for retrieving surgical data and patient data and a connection to the devices in the OR for automatic image acquisition and for automatic check-off of checklist tasks would be desirable. In addition to this, the times when the step was actually performed could be stored instead of the time when the checklist task was checked. Documentation of unforeseen events using for example free text fields or voice recordings would also be desirable.

\section{Author Statement}

Conflict of interest: Authors state no conflict of interest. Informed consent: This article does not contain patient data. Ethical approval: This article does not contain any studies with human participants or animals performed by the authors.

\section{References}

[1] Haynes AB, Weiser TG, Berry WR, Lipsitz SR, Breizat A-HS, Dellinger $E P$, et al. A surgical safety checklist to reduce morbidity and mortality in a global population. $\mathrm{N}$ Engl $\mathrm{J}$ Med. 2009;360:491-9.

[2] Vries EN de, Hollmann MW, Smorenburg SM, Gouma DJ, Boermeester MA. Development and validation of the
SURgical PAtient Safety System (SURPASS) checklist. Qual Saf Health Care. 2009;18:121-6

[3] Schmitz P, Strauss G. Effects of the Surgical Procedure Manager (SPM) on surgical procedure and planning. In: Abstract- und Posterband - 90. Jahresversammlung der Deutschen Gesellschaft für HNO-Heilkunde, Kopf- und HalsChirurgie e.V., Bonn - Digitalisierung in der HNO-Heilkunde; 29.05.2019 - 01.06.2019; Estrel Congress Center Berlin: Georg Thieme Verlag KG; $2019 .$.

[4] Ryniak C. Erstellung und prototypische Realisierung eines Konzepts zur Nutzung von Checklisten im OP am Beispiel der OR-Pad Anwendung [Masterthesis im Fach HumanCentered Computing zur]. Reutlingen: Hochschule Reutlingen; 25. 06.2019.

[5] Killer I. Entwicklung einer generischen Benutzeroberfläche zur Erfassung intraoperativer Prozessschritte und -zeiten [Bachelorarbeit zur Erlangung des akademischen Grades Bachelor of Science (B.Sc.)]. Reutlingen: Hochschule Reutlingen; 28.02.2020.

[6] Ryniak C, Burgert O. Automatic generation of checklists from business process model and notation (BPMN) models for surgical assist systems. Current Directions in Biomedical Engineering 2020.

[7] Wauben LSGL, van Grevenstein WMU, Goossens RHM, van der Meulen FH, Lange JF. Operative notes do not reflect reality in laparoscopic cholecystectomy. Br J Surg. 2011;98:1431-6.

[8] Miller D, Blanco E, Burns SK, Rao R, Stegmann R. System And Method Of Providing Visual Documentation During Surgery. United States Patent Application ublication. 2003.

[9] van de Graaf FW, Lange MM, Spakman JI, van Grevenstein WMU, Lips D, Graaf EJR de, et al. Comparison of Systematic Video Documentation With Narrative Operative Report in Colorectal Cancer Surgery. JAMA Surg. 2019;154:381-9. 